12

\title{
Топологические моды в неэрмитовых фононных кристаллах, индуцированных оптически в массиве квантовых ям
}

\author{
(C) А.В. Пошакинский, А.Н. Поддубный \\ Физико-технический инстиут им. А.Ф. Иофрфе, \\ Санкт-Петербург, Росси \\ E-mail: poshakinskiy@mail.ioffe.ru \\ Поступила в Редакцию 1 декабря 2020 г. \\ В окончательной редакции 1 декабря 2020 г. \\ Принята к публикации 3 декабря 2020 г.
}

\begin{abstract}
Изучено теоретически распространение акустических волн в массиве квантовых ям, в котором путем оптического возбуждения с частотой, близкой к экситонному резонансу, реализовано модулированное в пространстве усиление и ослабление звука. Рассчитана дисперсия звуковых волн вблизи брэгговских резонансов, соответствующих волновому вектору модуляции. Описано возникновение „мнимых“ стопзон, в которых происходит расщепление мнимых частей частоты блоховских мод. Продемонстрировано возникновение на краю структуры акустических топологических мод.
\end{abstract}

Ключевые слова: структуры с квантовыми ямами, акустические фононы, оптомеханика.

DOI: 10.21883/FTT.2021.04.50721.249

\section{1. Введение}

Простейшими одномерными системами, поддерживающими топологические краевые состояния, являются структуры, описываемые моделью Обри-АндреХарпера (Aubry-André-Harper, АAН) [1]. В этой модели рассматривается последовательность узлов, между которыми возможно туннелирование частиц, причем потенциал узла и/или константа туннелирования модулируется в зависимости от номера узла $j$ как $\cos (2 \pi \alpha j+\phi)$. Если $\alpha$ является рациональным числом, $\alpha=\mathscr{M} / \mathscr{N}$, где $\mathscr{M}$ и $\mathscr{N}$ целые, то такая структура является периодической, а ее элементарная ячейка содержит $\mathscr{N}$ узлов. При иррациональном $\alpha$ структура является квазикристаллом. В обоих случаях оказывается возможным установить соответствие между рассматриваемой одномерной системой и двумерной решеткой во внешнем магнитном поле, причем $\alpha$ играет роль магнитного потока через элементарную ячейку двумерной решетки (отнесенного к кванту магнитного потока) [2,3]. Поэтому энергетический спектр модели ААН, так же как и спектр двумерной системы в магнитном поле, может содержать зоны блоховских состояний с нетривиальной топологией, характеризующейся отличными от нуля числами Черна. В промежутках между такими зонами возникают краевые топологические моды. При изменении фазы модуляции $\phi$ от 0 до $2 \pi$ энергия топологической моды полностью заметает энергетический зазор между зонами.

Модель ААН, сформулированная изначально применительно к электронам, в настоящее время активно применяется для описания света в структурах, оптические свойства которых модулированы в пространстве. Ключевыми отличиями таких задач от канонической модели ААН является открытость и неэрмитовость. Радиационные потери, имеющие место в практически любой оптической системе, приводят к конечному времени жизни краевых топологических мод [4]. Такие излучающие краевые состояния проявляются в виде резонансных особенностей в спектрах отражения света от структуры, а по зависимости фазы коэффициента отражения от фазы модуляции $\phi$ можно определить числа Черна [5]. При наличии накачки возможно также реализовать оптическое усиление, модулированное в пространстве. Такая структура может быть описана неэрмитовой моделью ААН, где модуляция потенциалов узлов или констант туннелирования является комплексной величиной [6,7]. Топологическая классификация неэрмитовых моделей ААН гораздо богаче, чем канонических $[8,9]$. Краевые состояния могут быть стационарными, если накачка и потери сбалансированы. В обратном случае усиливающие краевые моды могут быть использованы для лазерной генерации [10]. Большое внимание в настоящее время привлекают также модели ААН за пределами одночастичного приближения, в которых взаимодействие возбуждений может приводить к топологическим фазовым переходам [11] и топологическим состояниям нескольких связанных частиц [12].

В настоящей работе рассмотрен теоретически массив квантовых ям, в котором путем оптического возбуждения с частотой, близкой к экситонному резонансу, реализовано модулированное в пространстве усиление и ослабление акустических волн, описываемое неэрмитовой моделью ААН. Рассчитана дисперсия звука в таком фононном кристалле, а также установлено существование в нем краевых топологических мод. 


\section{2. Модель}

\section{А. Взаимодействие звука с экситонами В квантовой яме}

В работе [13] нами было показано, что в квантовой яме, возбуждаемой интенсивным лазерным светом с частотой, близкой к экситонному резонансу, может реализовываться, в зависимости от знака отстройки, эффективное усиление или ослабление акустических волн. Эффект обусловлен резонансным поглощением или стимулированным излучением фононов при возбуждении экситона фотоном, энергия которого меньше или больше энергии экситона.

В настоящей работе будет рассмотрено возбуждение квантовых ям одновременно двумя электромагнитными волнами с частотами $\omega_{1}$ и $\omega_{2}$. Такое оптическое возбуждение индуцирует в квантовой яме экситоны на этих частотах с концентрациями $\left|b_{1}\right|^{2}$ и $\left|b_{2}\right|^{2}$,

$$
\left|b_{1,2}\right|^{2}=\frac{I_{1,2}}{\hbar \omega_{1,2}} \frac{\Gamma_{0}}{\Delta_{1,2}^{2}+\Gamma_{x}^{2}}
$$

где $b_{1,2}$ - когерентные амплитуды экситонов, $I_{1,2}-$ интенсивность света на частоте $\omega_{1,2}, \Delta_{1,2}=\omega_{1,2}-\omega_{x}-$ отстройки частот лазеров от экситонного резонанса, $\Gamma_{0}$ - радиационное затухание экситона, характеризующее силу его взаимодействия со светом, $\Gamma_{x}$ - нерадиационное затухание экситона, обусловленное потерями. Мы будем считать, что $\Gamma_{0} \ll \Gamma_{x}$, так что формирования гибридных фоноритонных мод $[14,15]$, обусловленных интенсивными процессами конверсии фононов в фотоны на стоксовой и анти-стоксовой частотах, не происходит.

Изменение акустических свойств квантовой ямы под действием оптического возбуждения, обусловленное взаимодействием возбужденных экситонов с фононами по механизму деформационного потенциала, описывается собственно-энергетической частью фонона $\Sigma$, играющей роль акустической восприимчивости квантовой ямы. Диаграммы процессов, вносящих вклад в $\Sigma$ во втором порядке по $b_{1,2}$, показаны на рис. 1 , где сплошные линии соответствуют экситонам, а волнистые - фононам. Собственно-энергетическая часть, описывающая рассеяние фонона без изменения его частоты $\Omega$, определяется диаграммами, изображенными на рис. $1, a, b$, и имеет вид [13]:

$$
\Sigma_{0}(\Omega)=\sum_{j=1,2} \frac{|\Xi|^{2}\left|b_{j}\right|^{2}}{2 \rho_{0} s_{0}^{3}} \frac{2 \Delta_{j} \Omega}{\Delta_{j}^{2}-\left(\Omega+i \Gamma_{x}\right)^{2}},
$$

где $\Xi-$ константа деформационного потенциала, $\rho_{0}$ и $s_{0}$ - плотность материала квантовой ямы и скорость продольного звука в ней. Наличие когерентного оптического возбуждения на одновременно двух частотах приводит к возможности изменения частоты фонона на $\delta \omega_{L}=\omega_{1}-\omega_{2}$. Амплитуда конверсии фонона с частотой $\Omega$ в фонон с частотой $\Omega+\delta \omega_{L}$ дается недиагональным

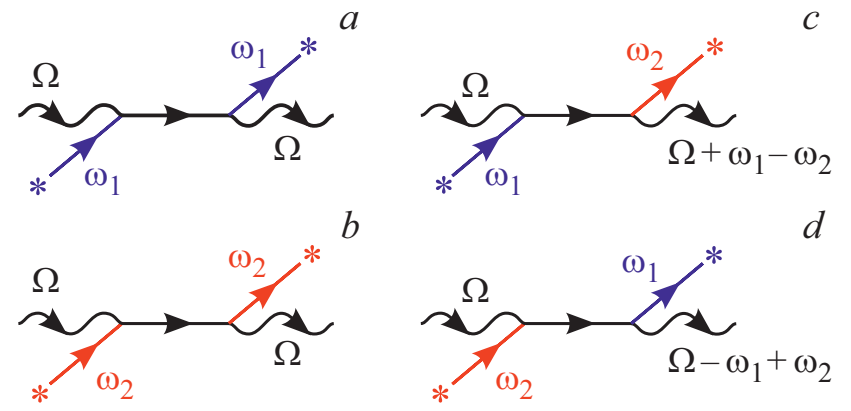

Рис. 1. Диаграммы процессов, вносящих вклад в акустическую восприимчивость квантовой ямы $\Sigma$ во втором порядке по амплитуде оптического возбуждения. Внешними прямыми линиями показаны амплитуды $b_{1,2}$ оптически созданных экситонов на частотах $\omega_{1,2}$. Внутренняя прямая линия соответствует экситонному пропагатору. Фононы обозначены волнистыми линиями. Вершины описывают взаимодействие экситонов с фононами по механизму деформационного потенциала.

элементом собственно-энергетической части, который соответствует диаграмме, изображенной на рис. $1, c$, и имеет вид

$$
\Sigma_{1}\left(\Omega+\delta \omega_{L}, \Omega\right)=\frac{|\Xi|^{2} b_{1} b_{2}^{*}}{2 \rho_{0} s_{0}^{3}} \frac{2\left(\bar{\omega}_{L}-\omega_{x}\right) \sqrt{\Omega\left(\Omega+\delta \omega_{l}\right)}}{\bar{\omega}_{L}^{2}-\left(\frac{\delta \omega_{L}}{2}+\Omega+i \Gamma_{x}\right)^{2}},
$$

где $\bar{\omega}_{L}=\left(\omega_{1}+\omega_{2}\right) / 2$. Амплитуда обратного процесса $\Sigma_{1}\left(\Omega-\delta \omega_{L}, \Omega\right)$ дается диаграммой, изображенной на рис. $1, d$, и может быть получена из уравнения (3) заменой $\omega_{1} \leftrightarrow \omega_{2}$.

Наличие процессов конверсии приводит к появлению бесконечной лестницы частот $\Omega_{m}=\Omega+m \delta \omega_{L}$, где $m$ - целое число. Переходы между модами $m$ и $m+1$ формально аналогичны туннелированию между соседними узлами в модели сильной связи, что позволяет рассматривать $m$ как дополнительное синтетическое измерение [16]. Однако если $\omega_{1}+\omega_{2}=2 \omega_{x}$, т.е. частоты двух возбуждающих лазеров расположены симметрично относительно частоты экситона, амплитуда конверсии (3) обращается в нуль. Именно такой случай, когда лестницы частот не возникает, будет рассмотрен в дальнейшем. В этом случае, коэффициенты прохождения и отражения звука, падающего на квантовую яму по нормали, могут быть выражены через акустическую восприимчивость (2):

$$
t(\Omega)=\frac{1}{1+i \Sigma_{0}(\Omega)}, \quad r(\Omega)=\frac{i \Sigma_{0}(\Omega)}{1+i \Sigma_{0}(\Omega)}
$$

Коэффициент усиления звука квантовой ямой определяется мнимой частью восприимчивости

$$
G(\Omega)=|t(\Omega)|^{2}+|r(\Omega)|^{2}-1=\frac{2 \operatorname{Im} \Sigma_{0}(\Omega)}{\left|1+i \Sigma_{0}(\Omega)\right|^{2}} .
$$




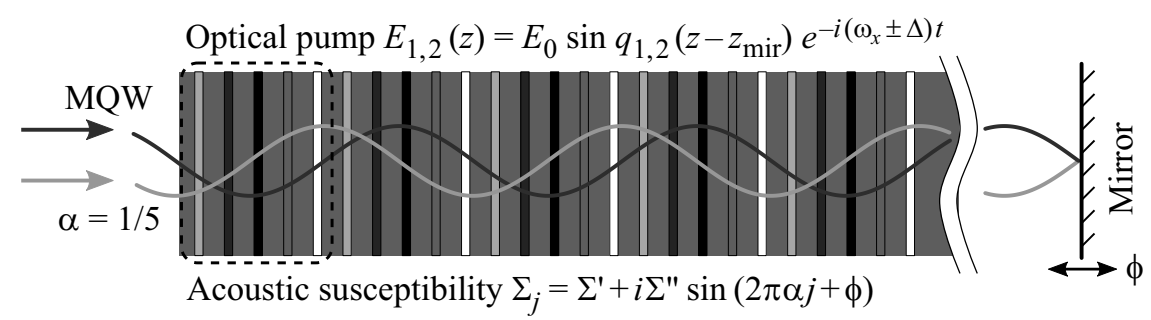

Рис. 2. Схема оптически индуцированного акустического кристалла. Оптическое возбуждение массива квантовых ям двумя стоячими электромагнитными волнами на частотах, отстроенных положительно и отрицательно от экситонного резонанса, приводит к пространственной модуляции акустической восприимчивости квантовых ям. Оттенками серого цвета показана мнимая часть акустической восприимчивости: черный цвет соответствует усилению звука, белый - поглощению. Показан случай, когда период модуляции в пять раз больше периода структуры, $\alpha=1 / 5$. Пунктиром выделена элементарная ячейка фононного кристалла.

\section{В. Метод матриц переноса для акустических волн}

Используя коэффициенты отражения и пропускания (4), можно построить матрицу переноса, связывающую амплитуды акустических волн на левой и правой границах квантовой ямы [17]. В базисе бегущих волн матрица переноса имеет вид

$$
\hat{T}_{\mathrm{QW}}\left(\Sigma_{0}\right)=\left[\begin{array}{cc}
1-i \Sigma_{0}(\Omega) & i \Sigma_{0}(\Omega) \\
-i \Sigma_{0}(\Omega) & 1+i \Sigma_{0}(\Omega)
\end{array}\right] .
$$

Помимо взаимодействия с оптически созданными экситонами, фононы также испытывают отражение от интерфейсов квантовой ямы, обусловленное различием механических свойств материала квантовой ямы и барьеров. Матрица переноса через интерфейс барьер/квантовая яма имеет вид [17]:

$$
\hat{T}_{\text {int }}(\beta)=\frac{1}{2}\left[\begin{array}{ll}
1+\beta & 1-\beta \\
1-\beta & 1+\beta
\end{array}\right],
$$

где $\beta=(\rho s) /\left(\rho_{0} s_{0}\right)$ характеризует отличие плотностей $\left(\rho_{0}\right.$ и $\left.\rho\right)$ и продольных скоростей звука $\left(s_{0}\right.$ и $\left.s\right)$ в материале квантовой ямы и барьере. Для квантовых ям GaAs/AlAs параметр $\beta \approx 0.84$. Матрица переноса через интерфейс квантовая яма/барьер дается $\hat{T}_{i n t}(1 / \beta)$.

Матрица переноса через квантовую яму и интерфесы определяется произведением $\hat{T}_{i n t}(1 / \beta) \hat{T}_{\mathrm{sp}}\left(k_{0} a / 2\right) \hat{T}_{\mathrm{QW}} \hat{T}_{\mathrm{sp}}\left(k_{0} a / 2\right) \hat{T}_{\mathrm{int}}(\beta)$, где $\hat{T}_{\mathrm{sp}}$ есть матрица переноса через однородную среду,

$$
T_{\mathrm{sp}}(\Phi)=\left[\begin{array}{cc}
e^{\mathrm{i} \Phi} & 0 \\
0 & e^{-i \Phi}
\end{array}\right],
$$

$k_{0}=\Omega / s_{0}-$ волновой вектор фонона внутри квантовой ямы, $a-$ ширина квантовой ямы. Мы будем рассматривать достаточно узкие квантовые ямы, для которых $k_{0} a \ll 1$. В этом случае матрица переноса через квантовую яму с интерфейсами принимает вид, схожий с уравнением (6), где $\Sigma_{0}$ необходимо заменить на $\Sigma$,

$$
\Sigma=\beta \sigma_{0}+\frac{k_{0} a\left(1-\beta^{2}\right)}{2 \beta} .
$$

Первое слагаемое в уравнении (9) описывает перенормировку силы взаимодействия звука с экситонами, обусловленную интерфейсами и аналогичную эффекту Парселла в оптике. Второе слагаемое представляет собой вещественный вклад в акустическую восприимчивость квантовой ямы и описывает отражение звука, обусловленное акустическим контрастом.

\section{С. Фононный кристалл на основе массива квантовых ям}

Мы рассматриваем периодическую структуру из $N$ квантовых ям, разделенных барьерами толщиной $d$, см. рис. 2. Структура возбуждается слева двумя лазерами на частотах $\omega_{1}=\omega_{x}+\Delta$ и $\omega_{2}=\omega_{x}-\Delta$ с одинаковой интенсивностью. Справа от системы находится зеркало, отражение от которого формирует внутри структуры стоячую электромагнитную волну. Интенсивность волны зависит от координаты вдоль оси роста $z$ как

$$
I_{1,2}(z)=I_{0} \sin ^{2}\left[q_{1,2}\left(z-z_{\mathrm{mir}}\right)\right],
$$

где $q_{1,2}=\omega_{1,2} / c-$ волновой вектор света, $z_{\text {mir }}-$ координата зеркала. Мы пренебрегли поглощением света на экситонном резонансе, которое дается формулой $2 N \Gamma_{0} \Gamma_{x} /\left(\Delta^{2}+\Gamma_{x}^{2}\right)$ и мало, если $\Gamma_{0} \ll \Gamma_{x}$, а частота лазера достаточно отстроена от резонанса. Согласно формуле (2), обусловленный оптическим возбуждением вклад в акустическую восприимчивость $j$-ой квантовой ямы, находящейся в точке $z_{j}=j d$, определяется разностью интенсивностей положительно и отрицательно отстроенного от частоты экситона электрического поля, $I_{1}\left(z_{j}\right)-I_{2}\left(z_{j}\right)$, и имеет вид

$$
\begin{aligned}
& \Sigma_{0}(\Omega)=\frac{|\Xi|^{2} \Gamma_{0} I_{0}}{\rho_{0} s_{0}^{3} \omega_{L}\left(\Delta^{2}+\Gamma_{x}^{2}\right)} \frac{\Omega \Delta}{\Delta^{2}-\left(\Omega+\mathrm{i} \Gamma_{x}\right)^{2}} \\
& \quad \times \sin \left[\left(q_{1}-q_{2}\right)\left(z_{j}-z_{\mathrm{mir}}\right)\right] \sin \left[\left(q_{1}+q_{2}\right)\left(z_{j}-z_{\mathrm{mir}}\right)\right] .
\end{aligned}
$$

Разностью волновых векторов $q_{1}-q_{2}=2 \Delta / c$ можно пренебречь на масштабах длины структуры $N d$, если период структуры $d \sim s / \Delta$, а число квантовых ям 
$N \ll c / s \sim 10^{5}$. Тогда первый синус в зависимости (11) равен константе, и вся координатная зависимость определяется $\sin \left[2 q_{L}\left(z_{j}-z_{\text {mir }}\right)\right]$.

Мы будем интересоваться свойствами звука, частота которого близка к отстройке лазеров от экситонного резонанса, $\Omega \approx \Delta$. В этом случае влияние оптического возбуждения на акустические свойства, описываемое уравнением (11), максимально. Полная собственноэнергетическая часть, уравнение $(9)$, для $j$-ой квантовой ямы принимает вид

$$
\Sigma_{j}=\Sigma^{\prime}+\mathrm{i} \Sigma^{\prime \prime} \sin (2 \pi \alpha j+\phi),
$$

где $\alpha=q_{L} d / \pi$ определяется отношением периода структуры $d$ к длине волны света лазера, фаза $\phi=-2 q_{L} z_{\text {mir }}$ контролируется положением зеркала, $\Sigma^{\prime}=k_{0} a\left(1-\beta^{2}\right) /(2 \beta)$ определяется акустическим контрастом, а $\Sigma^{\prime \prime}=|\Xi|^{2} \Gamma_{0} I_{0} /\left(2 \rho_{0} s_{0}^{3} \omega_{L} \Delta \Gamma_{x}\right)$ обусловлено оптической накачкой. Для реалистичных массивов квантовых ям $\mathrm{GaAs} / \mathrm{AlGaAs}$, подобных изученным в работе $[18], \quad \Sigma^{\prime} \sim 0.4$, а $\Sigma^{\prime} \sim 0.05$ для накачки с интенсивностью $0.1 \mathrm{~mW} / \mu \mathrm{m}^{2}$, соответствующей концентрации экситонов $\left|b_{L}\right|^{2} \sim 10^{10} \mathrm{~cm}^{-2}[13]$.

Гармоническая модуляция акустических свойств (12) имеет вид, аналогичный модуляции энергий узлов в модели ААН. Ключевым отличием является то, что в рассматриваемой задаче амплитуда модуляции чисто мнимая. Модулируется не вещественная, а мнимая часть акустического импеданса, т. е. коэффициент поглощения и усиления звука.

\section{3. Дисперсия звука и краевые акустические моды}

\section{А. Дисперсия звука}

Для простоты мы будем считать, что длина волны лазера соразмерна с расстоянием между квантовыми ямами $d$, т.е. $\alpha$ рационально, $\alpha=\mathscr{M} / \mathscr{N}$, где $\mathscr{M}$ и $\mathscr{N}$ - целые числа. Тогда период структуры с учетом оптической модуляции увеличивается до $D=d \mathscr{N}$. Акустическая матрица переноса через период дается выражением

$$
\hat{T}^{(1)}=\prod_{j=1}^{\mathscr{N}} \hat{T}_{\mathrm{sp}}(k d) \hat{T}_{\mathrm{QW}}\left(\Sigma_{j}\right)
$$

где при вычислении произведения множители должны располагаться справа налево в порядке увеличения $j$. Учитывая, что в реальных системах $\Sigma_{j} \ll 1$, матрицу $\hat{T}^{(1)}$ можно вычислить в линейном порядке по $\Sigma_{j}$, что дает

$$
\hat{T}^{(1)}=\left[\begin{array}{cc}
\left(1-\mathrm{i} f_{0}\right) e^{\mathrm{i} k D} & \mathrm{i} f_{k} e^{\mathrm{i} k D} \\
-\mathrm{i} f_{-k} e^{-\mathrm{i} k D} & \left(1+\mathrm{i} f_{0}\right) e^{-\mathrm{i} k D}
\end{array}\right] .
$$

Здесь $f_{k}-$ структурный фактор, представляющий собой дискретное преобразование Фурье от $\Sigma_{j}$ внутри элементарной ячейки,

$$
f_{k}=\sum_{j=1}^{\mathscr{N}} \Sigma_{j} e^{-2 \mathrm{i} k z_{j}}
$$

Приравнивая собственные числа матрицы переноса через элементарную ячейку (14) величине $e^{ \pm \mathrm{i} K D}$, можно определить блоховский волновой вектор $K$, описывающий распространение звука в бесконечной периодической структуре. Закон дисперсии блоховских мод, т.е. связь между блоховским волновым вектором $K$ и частотой звука $\Omega=s k$, имеет вид

$$
\sin ^{2} K D=\sin ^{2}\left(k D-f_{0}\right)-f_{k} f_{-k} .
$$

Если $f_{-k}= \pm f_{k}^{*}$, т.е. $f_{k}$ вещественное или чисто мнимое, дисперсионное уравнение (16) представляет собой аналитическую функцию с вещественными коэффициентами, а значит его решения либо вещественны, либо представляют собой пары комплексно сопряженных чисел.

Вдали от границ зоны Биллюэна вторым слагаемым в правой части уравнения (16), которое квадратично по $\Sigma_{j}$, можно пренебречь, и дисперсия звука принимает простую форму $K=\Omega / s-f_{0} / D$. Этот результат соответствует перенормировке скорости звука в слоистой структуре, вследствие усреднения акустических свойств (гармоника $f_{0}$ представляет собой усредненный акустический контраст).

Вблизи частот $\Omega_{g}=(s / D)\left(g \pi+f_{0}\right)$, где $g$ - целое, дисперсия акустических волн сильно модифицируется вследствие брэгговской дифракции. Линеаризуя уравнение (16) вблизи таких частот, получаем

$$
\tilde{\Omega}^{2}=s^{2} \tilde{K}^{2}+\delta^{2},
$$

где

$$
\delta^{2}=(s / D)^{2} f_{g \pi / D} f_{-g \pi / D},
$$

$\tilde{\Omega}=\Omega-\Omega_{g}$ и $\tilde{K}=K-g \pi / D-$ отстройка частоты и волнового вектора от величин, соответствующих условию Брэгга. В случае вещественного и положительного $\delta^{2}$ уравнение (17) описывало бы дираковскую дисперсию, в которой $\delta$ играет роль массы и приводит к появлению акустической стоп-зоны шириной $2 \delta$ при $\tilde{K}=0$.

В рассматриваемом нами неэрмитовом фононном кристалле с модуляцией акустического поглощения и усиления, описываемой уравнением (12), отличные от нуля компоненты структурного фактора имеют вид

$$
\begin{gathered}
f_{0}=\mathscr{N} \Sigma^{\prime}, \\
f_{ \pm \pi \mathscr{M} / D}= \pm \mathscr{N} e^{ \pm \mathrm{i} \phi} \Sigma^{\prime \prime} / 2 .
\end{gathered}
$$

Вблизи частот $\Omega \approx s n \mathscr{N} \pi / D$, где $n-$ целое, дисперсия звука описывается уравнением (17) с вещественным 


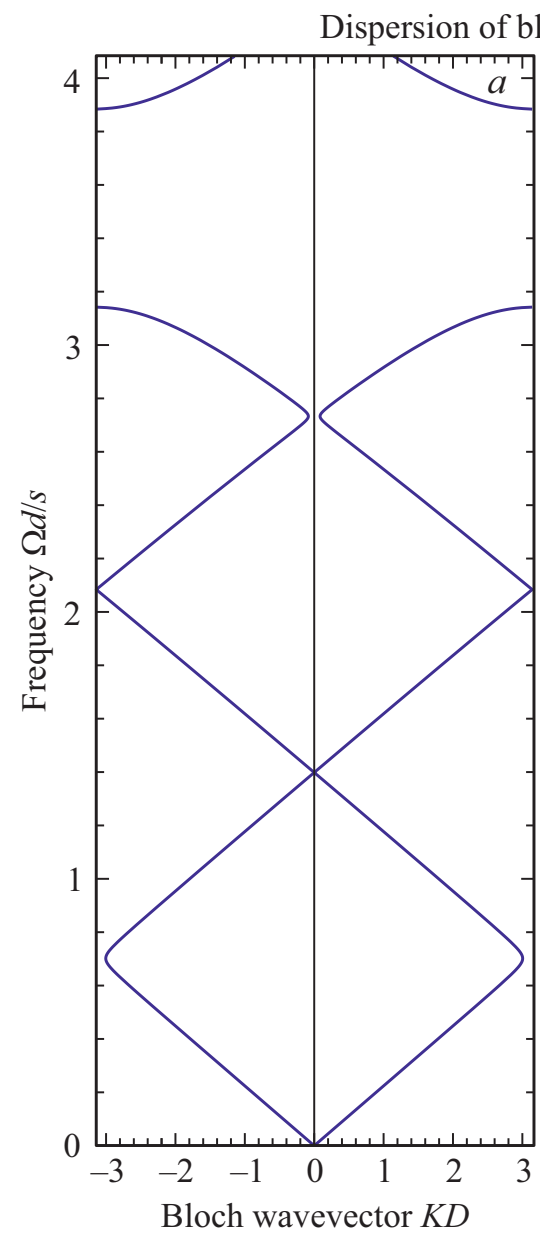

ch modes

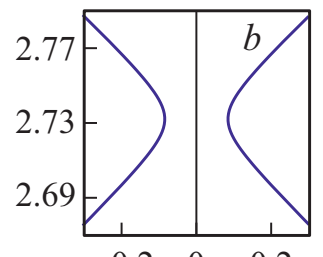

$\begin{array}{lll}-0.2 & 0 & 0.2\end{array}$
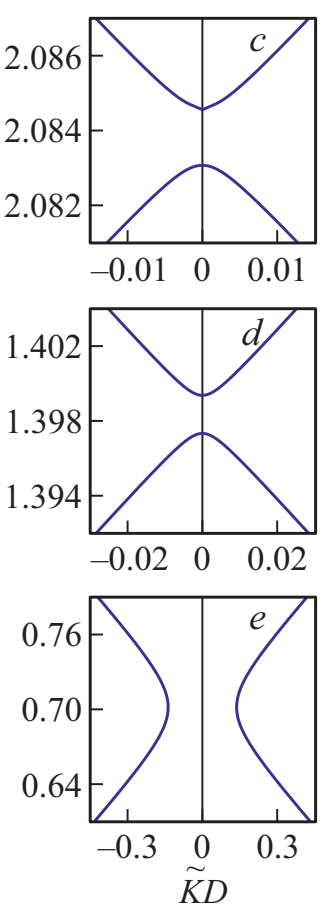

Рис. 3. (a) Дисперсия звука в оптически индуцированном неэрмитовом фононном кристалле на основе массива квантовых ям, акустическая восприимчивость которых описывается формулой (12) с $\alpha=1 / 5, \phi=0, \Sigma^{\prime}=0.1 \Omega d / s, \Sigma^{\prime \prime}=0.05$. Панели $(b-e)$ показывают в более крупном масштабе дисперсию звука вблизи четырех брэгговских резонансов, обусловленных оптической модуляцией.

$\delta=\Sigma^{\prime} s / d$. Вблизи частот $\Omega \approx s \pi(n \mathscr{N} \pm \mathscr{M}) / D$ дисперсия звука описывается уравнением (17) с „чисто мнимой“ стоп-зоной $\delta=\mathrm{i} \Sigma^{\prime \prime} s /(2 d)$. В этом случае частоты собственных мод в диапазоне $|\tilde{K}|<\delta / s$ являются комплексными, а расщепление между ними - чисто мнимым. Одна из собственных частот имеет положительную мнимую часть, т.е. мода нарастает со временем, а другая - отрицательную, т.е. мода затухает со временем. Физически, наличие нарастающей моды означает возможность акустической лазерной генерации. Генерация начинается, когда мнимая часть частоты моды превосходит затухание, обусловленное неучтенными в нашей модели, но всегда присутствующими в реальной системе акустическими потерями. Вдали от брэгговского резонанса частоты обоих мод мод вещественны, что обусловлено тем, что накачка и поглощение звука в рассматриваемом фононном кристалле в среднем скомпенсированы.
На рис. 3, а показана дисперсия звука в фононном кристалле с $\alpha=1 / 5$. Широкая стоп-зона на частотах (измеряемых в единицах $s / d$ ) от 3.2 до 3.8 обусловлена акустическим контрастом между материалом ямы и барьеров, который описывается величиной $\Sigma^{\prime}=0.35$. Оптическая накачка, приводящая к модуляции акустического усиления и поглощения с периодом в пять раз большим, чем период массива квантовых ям, приводит к появлению еще четырех брэгговских резонансов на более низких частотах, интенсивность которых определяется величиной $\Sigma^{\prime \prime}=0.05$. Дисперсия вблизи этих резонансов показана на панелях рис. $3, b-e$. Для брэгговских резонансов вблизи частот 0.70 и 2.73 , см. рис. $3, b, e$, описываемых отличными от нуля структурными факторами $f_{ \pm \pi /(5 d)}, \delta$ чисто мнимое, что приводит к появлению „мнимых“ стоп-зон. Структурные факторы $f_{ \pm 2 \pi /(5 d)}$, соответствующие брэгговским резонансам вблизи частот 1.398 и 2.084, для рассматриваемой модуляции равны нулю. Брэгговская дифракция возможна лишь во втором порядке по $\Sigma^{\prime \prime}$. В результате в этих точках возникают узкие реальные стоп-зоны, описываемые вещественным $\delta$, см. рис. $3, c, d$.

\section{В. Акустические топологические состояния}

При изменении фазы модуляции $\phi$ от $-\pi$ до $\pi$ структурный фактор $f_{ \pm \pi \mathscr{M} / D} \sim e^{ \pm \mathrm{i} \phi}$ делает в комплексной плоскости один полный оборот вокруг начала координат. Отличное от нуля число намоток структурного фактора $w= \pm 1$ свидетельствует о нетривиальной топологии блоховских состояний и должно приводить к возникновению $|w|$ краевых топологических мод, которые при изменении $\phi$ пересекают соответствующую стоп-зону в направлении, определяемом знаком $w$. Наличие краевых мод топологически защищено от модификаций границы структуры: при добавлении на границу произвольных дополнительных слоев краевые моды сохраняются, хотя диапазон $\phi$, в котором они существуют, может измениться [5].

Частоты мод, локализованных на левом краю полубесконечного массива, могут быть найдены из уравнений для компонент матрицы переноса через период [4]

$$
\hat{T}_{12}^{(1)}(\Omega)=0, \quad\left|\hat{T}_{22}^{(1)}(\Omega)\right|<1 .
$$

На рис. 4, $a-d$ сплошными кривыми показаны рассчитанные зависимости вещественной части частоты краевого состояния от фазы модуляции. Как и ожидалось исходя из чисел намотки структурного фактора $w= \pm 1$, состояния на рис. $4, a, d$ пересекают частоту соответствующей „мнимой“ стоп-зоны, обозначенную пунктиром, один раз, но в противоположных направлениях. Краевые состояния на рис. 4, b, $c$ пересекают частоту, соответствующую реальной стоп-зоне, два раза, и также в противоположных направлениях. Более высокие числа намотки $w= \pm 2$ объясняются тем, что смешивание зон в данных брэгговских резонансах происходит лишь во 
Edge state frequency
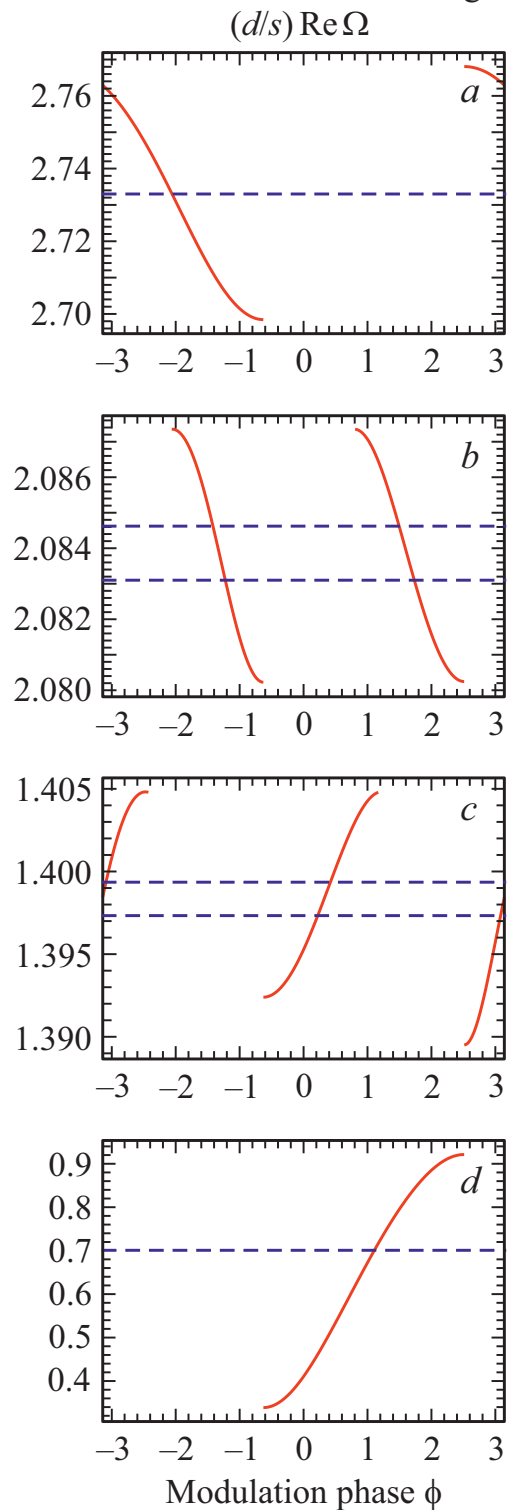

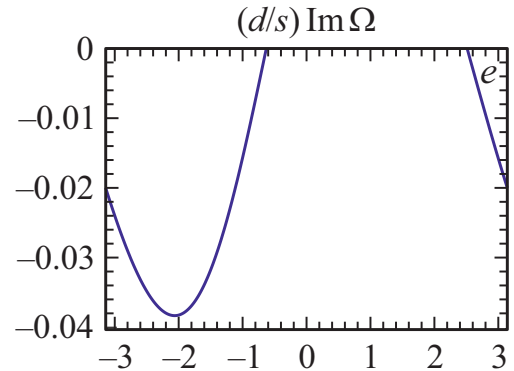

Edge state spatial decay
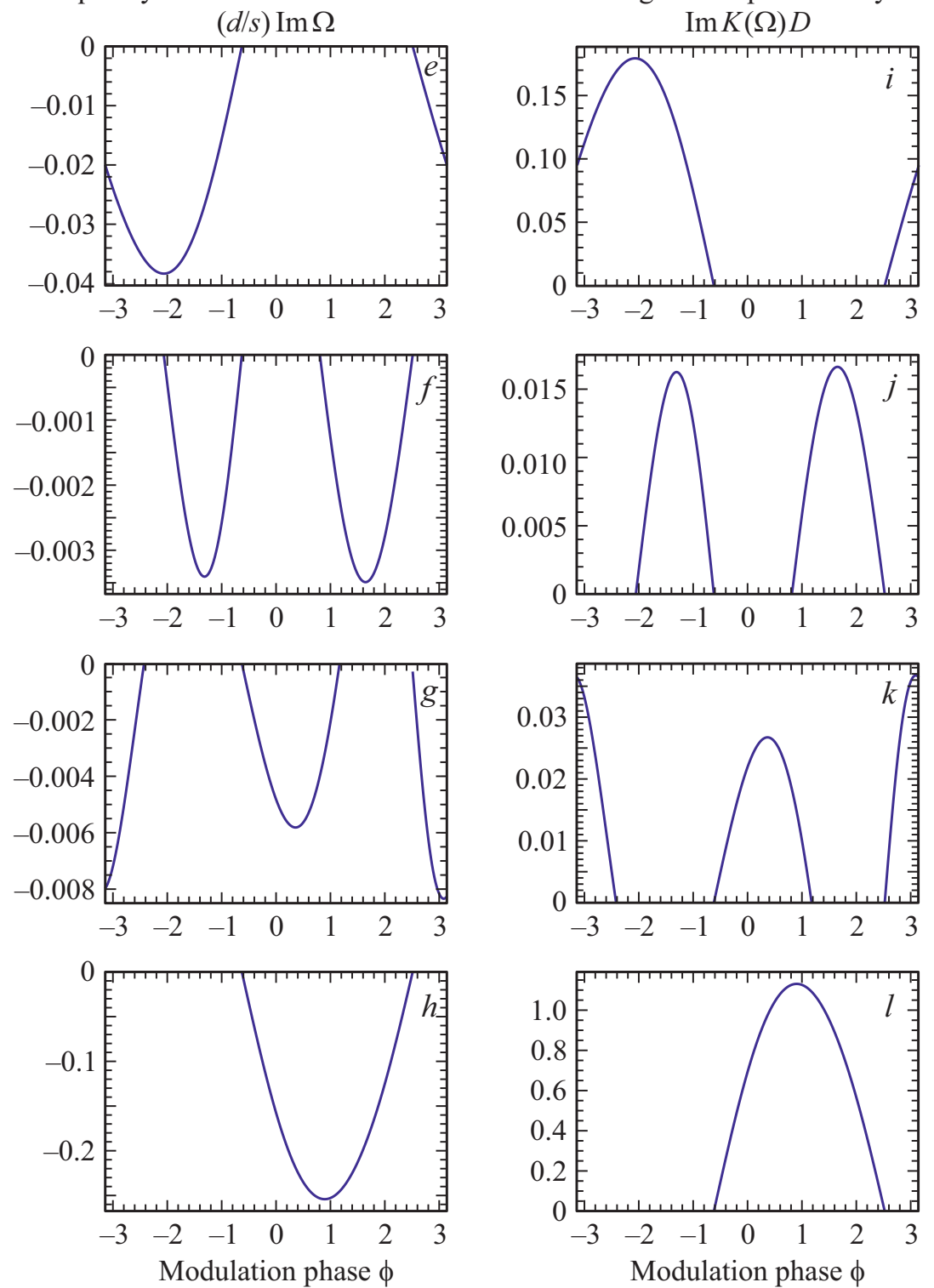

Рис. 4. $(a)-(d)$ Частоты акустических краевых мод, возникающих вблизи четырех брэгговских резонансов, обусловленных оптической модуляцией, как функция фазы модуляции. Пунктиром показаны границы реальных стоп-зон и частоты „мнимых“ стоп-зон. $(e-h)$ Мнимая часть частоты краевой моды как функция фазы модуляции. $(i)-(l)$. Пространственная скорость затухания краевых мод вглубь структуры как функция фазы модуляции. Расчет выполнен для тех же параметров, что рис. 3

втором порядке теории возмущений и пропорционально $f_{ \pm \pi /(5 d)}^{2} \sim e^{ \pm 2 \mathrm{i} \phi}$.

На рис. 4, $e-h$ показаны рассчитанные зависимости мнимой части частоты краевого состояния от фазы модуляции. Несмотря на то, что в структуре есть как квантовые ямы, усиливающие звук, так и ямы, его ослабляющие, мнимая часть частоты краевого состояния является всегда отрицательной, т.е. краевое состояние является всегда затухающим, в отличие от объемных блоховских мод, которые могут как затухать, так и возрастать. Это обусловлено тем, что доминирующий вклад в мнимую часть энергии краевого состояния вносят радиационные потери на границе, вблизи которой оно локализовано. Обратная глубина локализации краевых состояний, определяемая величиной $-\ln \left|\hat{T}_{22}^{(1)}(\Omega)\right|$, построена на рис. $4, i-l$. Чем более состояние делокализовано, тем ближе к нулю мнимая часть его частоты.

\section{4. Заключение}

Нами предложен способ реализации неэрмитовой модели Обри-Андре-Харпера (Aubry-André-Harper, ААН) для звука, основанный на оптической модуляции 
акустических свойств массива квантовых ям стоячей электромагнитной волной. Амплитуда модуляции определяется интенсивностью света, а фазой модуляции можно управлять, меняя положение зеркала, формирующего стоячую волну. Показано, что на краю структуры возникают акустические топологические моды, частота которых пересекает акустические стоп-зоны при сканировании фазы модуляции. Краевые моды обладают конечным радиационным временем жизни, обусловленным уходом звука через границу системы.

\section{Финансирование работы}

Работа выполнена при поддержке гранта Российского научного фонда № 20-12-00194.

\section{Конфликт интересов}

Авторы заявляют, что у них нет конфликта интересов.

\section{Список литературы}

[1] S. Aubry, G. Andre. Ann. Isr. Phys. Soc. 3, 133 (1980).

[2] L.-J. Lang, X. Cai, S. Chen. Phys. Rev. Lett. 108, 220401 (2012).

[3] Y.E. Kraus, Y. Lahini, Z. Ringel, M. Verbin, O. Zilberberg. Phys. Rev. Lett. 109, 106402 (2012).

[4] A.V. Poshakinskiy, A.N. Poddubny, L. Pilozzi, E.L. Ivchenko. Phys. Rev. Lett. 112, 107403 (2014).

[5] A.V. Poshakinskiy, A.N. Poddubny, M. Hafezi. Phys. Rev. A 91, 043830 (2015).

[6] A.K. Harter, T.E. Lee, Y.N. Joglekar. Phys. Rev. A 93, 062101 (2016).

[7] Q.-B. Zeng, S. Chen, R. Lu. Phys. Rev. A 95, 062118 (2017).

[8] S. Longhi. Phys. Rev. Lett. 122, 237601 (2019).

[9] Q.-B. Zeng, Y.-B. Yang, Y. Xu. Phys. Rev. B 101, 020201 (2020).

[10] L. Pilozzi, C. Conti. Phys. Rev. B 93, 195317 (2016).

[11] D.-W. Zhang, Y.-L. Chen, G.-Q. Zhang, L.-J. Lang, Z. Li, S.-L. Zhu. Phys. Rev. B 101, 235150 (2020).

[12] Y. Ke, J. Zhong, A.V. Poshakinskiy, Y.S. Kivshar, A.N. Poddubny, C. Lee. Phys. Rev. Res. 2, 033190 (2020).

[13] A.V. Poshakinskiy, A.N. Poddubny, A. Fainstein. Phys. Rev. Lett. 117, 224302 (2016).

[14] A.L. Ivanov, L. Keldysh. Zh. Eksp. Teor. Fiz. 84, 404 (1982), [Sov. Phys. JETP 57, 234 (1983)].

[15] A.V. Poshakinskiy, A.N. Poddubny. Phys. Rev. Lett. 118, 156801 (2017)

[16] L. Yuan, Q. Lin, M. Xiao, S. Fan. Optica 5, 1396 (2018).

[17] E.L. Ivchenko. Optical Spectroscopy of Semiconductor Nanostructures. Alpha Science International, Harrow, UK (2005).

[18] B. Jusserand, A.N. Poddubny, A.V. Poshakinskiy, A. Fainstein, A. Lemaitre. Phys. Rev. Lett. 115, 267402 (2015).

Редактор Е.Ю. Флегонтова 